\title{
CODI-CRAC 2021
}

\section{The CODI-CRAC 2021 Shared Task on \\ Anaphora, Bridging, and Discourse Deixis in Dialogue}

Proceedings of the Workshop

November 10, 2021

Punta Cana, Dominican Republic 
C2021 The Association for Computational Linguistics

Order copies of this and other ACL proceedings from:

Association for Computational Linguistics (ACL)

209 N. Eighth Street

Stroudsburg, PA 18360

USA

Tel: +1-570-476-8006

Fax: +1-570-476-0860

acleaclweb.org

ISBN 978-1-955917-08-7 


\section{Message from the Program Chairs}

This shared task represents the collaborative efforts of a diverse team of researchers, across countries, institutional types, research areas, and career stages. The idea emerged through a Discourse Analysis breakout session at SIGDIAL 2020. The goal was to foster a more active partnership between the discourse and dialogue communities and to offer, not just a competition, but an opportunity to identify the next great challenges for the area of coreference.

Since that initial discussion at the Spring 2020 conference, the core organizing team has met almost weekly to build a vision for this shared task as the initial event out of what is hoped to become a series of such events, and situated in partnership with other related efforts, such as the Universal Anaphora effort. The team is excited to host this shared task, jointly with the CODI and CRAC workshops, co-located with EMNLP 2021. Each of the organizing team's members are also grateful to their respective institutions for providing the kind of environment that facilitates such international collaborations.

The team is grateful for funding committed by the Heidelberg Institute for Theoretical Studies and the Dali Project at Queen Mary University (both of which supported annotation for this shared task event) and Carnegie Mellon University (slated to support annotation for next year's event), as well as resources provided by Intel (especially in connection with the CODALAB infrastructure). The annotation team managed by Queen Mary University worked tirelessly to produce the annotated data, without which this shared task would not be a shared task at all! The team is also grateful for the synergistic efforts of the broader ACL community, for providing an environment in which the vision for this shared task could be realized and situated within this vibrant EMNLP milieu. 



\section{Organizing Committee}

- Sopan Khosla, Amazon, USA

- Ramesh Manuvinakurike, Intel, USA

- Vincent Ng, University of Texas at Dallas, USA

- Massimo Poesio, Queen Mary University, UK

- Michael Strube, Heidelberg Institute for Theoretical Studies, Germany

- Carolyn Rosé, Carnegie Mellon University, USA 



\section{Table of Contents}

The CODI-CRAC 2021 Shared Task on Anaphora, Bridging, and Discourse Deixis in Dialogue

Sopan Khosla, Juntao Yu, Ramesh Manuvinakurike, Vincent Ng, Massimo Poesio, Michael Strube and Carolyn Rosé .....................................................

Neural Anaphora Resolution in Dialogue

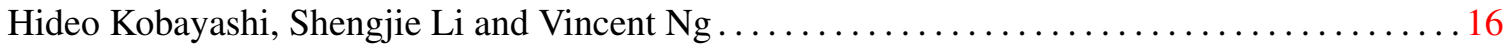

Anaphora Resolution in Dialogue: Description of the DFKI-TalkingRobots System for the CODI-CRAC 2021 Shared-Task

Tatiana Anikina, Cennet Oguz, Natalia Skachkova, Siyu Tao, Sharmila Upadhyaya and Ivana

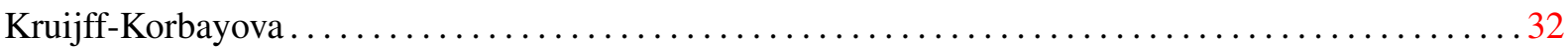

The Pipeline Model for Resolution of Anaphoric Reference and Resolution of Entity Reference

Hongjin Kim, Damrin Kim and Harksoo Kim.................................. 43

An End-to-End Approach for Full Bridging Resolution

Joseph Renner, Priyansh Trivedi, Gaurav Maheshwari, Rémi Gilleron and Pascal Denis ....... 48

Adapted End-to-End Coreference Resolution System for Anaphoric Identities in Dialogues

Liyan Xu and Jinho D. Choi ......................................... 55

Anaphora Resolution in Dialogue: Cross-Team Analysis of the DFKI-TalkingRobots Team Submissions for the CODI-CRAC 2021 Shared-Task

Natalia Skachkova, Cennet Oguz, Tatiana Anikina, Siyu Tao, Sharmila Upadhyaya and Ivana

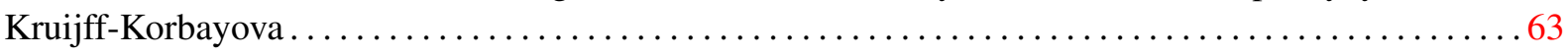

The CODI-CRAC 2021 Shared Task on Anaphora, Bridging, and Discourse Deixis Resolution in Dialogue: A Cross-Team Analysis

Shengjie Li, Hideo Kobayashi and Vincent Ng . .71 



\section{Workshop Program}

Wednedsday, November 10, 2021

\section{9:05-09:30 Session 1: Welcome}

09:05-09:30 The CODI-CRAC 2021 Shared Task on Anaphora, Bridging, and Discourse Deixis in Dialogue

Sopan Khosla, Juntao Yu, Ramesh Manuvinakurike, Vincent Ng, Massimo Poesio, Michael Strube and Carolyn Rosé

\section{9:30-10:30 Session 2: System Talks (part 1)}

09:30-09:45 Neural Anaphora Resolution in Dialogue

Hideo Kobayashi, Shengjie Li and Vincent Ng

09:45-10:00 Anaphora Resolution in Dialogue: Description of the DFKI-TalkingRobots System for the CODI-CRAC 2021 Shared-Task

Tatiana Anikina, Cennet Oguz, Natalia Skachkova, Siyu Tao, Sharmila Upadhyaya and Ivana Kruijff-Korbayova

10:00-10:15 The Pipeline Model for Resolution of Anaphoric Reference and Resolution of Entity Reference

Hongjin Kim, Damrin Kim and Harksoo Kim

10:15-10:30 An End-to-End Approach for Full Bridging Resolution

Joseph Renner, Priyansh Trivedi, Gaurav Maheshwari, Rémi Gilleron and Pascal Denis

\section{0:30-11:00 Coffee Break}

\section{1:00-11:15 Session 3: System Talks (part 2)}

11:00-11:15 Adapted End-to-End Coreference Resolution System for Anaphoric Identities in Dialogues

Liyan $\mathrm{Xu}$ and Jinho D. Choi 
Wednedsday, November 10, 2021 (continued)

\section{1:15-11:45 Session 4: Analysis Papers}

11:15-11:30 Anaphora Resolution in Dialogue: Cross-Team Analysis of the DFKI-TalkingRobots Team Submissions for the CODI-CRAC 2021 Shared-Task

Natalia Skachkova, Cennet Oguz, Tatiana Anikina, Siyu Tao, Sharmila Upadhyaya and Ivana Kruijff-Korbayova

11:30-11:45 The CODI-CRAC 2021 Shared Task on Anaphora, Bridging, and Discourse Deixis Resolution in Dialogue: A Cross-Team Analysis

Shengjie Li, Hideo Kobayashi and Vincent $\mathrm{Ng}$

11:45-12:00 Session 5: Visioning and Discussion 